\title{
A Classification of Sub-Riemannian Structures on the Heisenberg Groups
}

\section{Rory Biggs}

Department of Mathematics (Pure and Applied), Rhodes University, PO Box 94, 6140 Grahamstown, South Africa rorybiggs@gmail.com

\section{Péter T. Nagy}

Institute of Applied Mathematics, Óbuda University, H-1034 Budapest, Bécsi út 96/b, Hungary

nagy.peter@nik.uni-obuda.hu

\begin{abstract}
We apply Williamson's theorem for the diagonalization of quadratic forms by symplectic matrices to sub-Riemannian (and Riemannian) structures on the Heisenberg groups. A classification of these manifolds, under isometric Lie group automorphisms, is obtained. A (parametrized) list of equivalence class representatives is identified; a geometric characterization of this equivalence relation is provided. A corresponding classification of (drift-free) invariant optimal control problems is exhibited.
\end{abstract}

Keywords: Heisenberg group; sub-Riemannian geometry; isometry; symplectic group; invariant optimal control; cost-equivalence

\section{Introduction}

Riemannian geometry is concerned with the (higher dimensional theory of) metric geometry of Euclidean surfaces and in particular the length-minimizing curves on these surfaces. Sub-Riemannian geometry may be interpreted as a generalization of Riemannian geometry. The fundamental difference is that for a sub-Riemannian manifold motion is restricted to certain admissible (or horizontal) directions. Due to such constraints it may not be possible, in general, to connect any two points by a (horizontal) curve. Sub-Riemannian geometry has been a full research domain since the 1980's; it has motivations and ramifications in several areas of pure and applied mathematics. Moreover, there is a substantial overlap between sub-Riemannian geometry $([7,16])$, geometric optimal control $([2,12,18])$ and nonholonomic mechanics $([5,8])$. 
Among the sub-Riemannian manifolds, the Carnot groups are the most fundamental. In the words of Montgomery [16] "Carnot groups are to sub-Riemannian geometry as Euclidean spaces are to Riemannian geometry." The Heisenberg groups are the simplest, non-Euclidean Carnot groups. Structures on the Heisenberg groups (and their generalizations) have been extensively studied in the last few decades (see, e.g., $[4,9,14,15,19])$.

In this paper we shall classify, under isometric Lie group automorphisms, the leftinvariant bracket-generating sub-Riemannian (and Riemannian) structures on the $(2 n+1)$-dimensional (polarized) Heisenberg group

$$
\mathrm{H}_{n}=\left\{\left[\begin{array}{cccccc}
1 & x_{1} & x_{2} & \cdots & x_{n} & z \\
0 & 1 & 0 & & 0 & y_{1} \\
0 & 0 & 1 & & 0 & y_{2} \\
\vdots & & & \ddots & & \vdots \\
0 & & \cdots & & 1 & y_{n} \\
0 & & \cdots & & 0 & 1
\end{array}\right]: x_{i}, y_{i}, z \in \mathbb{R}\right\}
$$

$\mathrm{H}_{n}$ is a simply-connected two-step nilpotent Lie group with one-dimensional center; its Lie algebra

$$
\mathfrak{h}_{n}=\left\{\left[\begin{array}{cccccc}
0 & x_{1} & x_{2} & \cdots & x_{n} & z \\
0 & 0 & 0 & & 0 & y_{1} \\
0 & 0 & 0 & & 0 & y_{2} \\
\vdots & & & \ddots & & \vdots \\
0 & & \cdots & & 0 & y_{n} \\
0 & & \cdots & & 0 & 0
\end{array}\right]=z Z+\sum_{i=1}^{n}\left(x_{i} X_{i}+y_{i} Y_{i}\right): x_{i}, y_{i}, z \in \mathbb{R}\right\}
$$

has non-zero commutators $\left[X_{i}, Y_{j}\right]=\delta_{i j} Z$. Moreover, any simply-connected twostep nilpotent Lie group with one-dimensional center is isomorphic to $\mathrm{H}_{n}$.

Let us fix a sub-Riemannian structure on $\mathrm{H}_{n}$. A standard computation yields the automorphism group of $\mathrm{H}_{n}$, a subgroup of which is a symplectic group. By use of the automorphisms, we normalize the distributions on $\mathrm{H}_{n}$. Equivalence class representatives are then constructed by successively applying automorphisms, that preserve the normalized distribution, to the metric. (The Riemannian case is treated similarly.) Central to our argument is Williamson's theorem, which states that any positive definite symmetric matrix can be diagonalized, in a certain way, by symplectic matrices. Furthermore, we shall characterize (in coordinate-free form) when two sub-Riemannian (resp. Riemannian) structures on $\mathrm{H}_{n}$ are equivalent. (This characterization is based on decomposing $\mathfrak{h}_{n}$, as a vector space, into the product of a symplectic vector space and $\mathbb{R}$.)

To every invariant sub-Riemannian (resp. Riemannian) structure we can naturally associate an invariant optimal control problem (cf. [18]). Accordingly, a classification of sub-Riemannian and Riemannian structures may induce a classification of invariant optimal control problems (or rather, cost-extended systems). In the last section, we exhibit the corresponding classification of invariant optimal control problems on $\mathrm{H}_{n}$. 


\subsection{Left-Invariant Sub-Riemannian Structures}

By a left-invariant sub-Riemannian manifold, we mean a triplet $(G, \mathscr{D}, \mathbf{g})$, where $\mathrm{G}$ is a (real, finite dimensional) connected Lie group with unit element $\mathbf{1}, \mathscr{D}$ is a smooth left-invariant distribution on $\mathbf{G}$, and $\mathbf{g}$ is a left-invariant Riemannian metric on $\mathscr{D}$. More precisely, $\mathscr{D}(\mathbf{1})$ is a linear subspace of the Lie algebra $\mathfrak{g}$ of $\mathrm{G}$ which is left-translated to the tangent bundle $T G$ via

$\mathscr{D}(g)=g \mathscr{D}(\mathbf{1}) \quad$ for $\quad g \in \mathrm{G}$.

The metric $\mathbf{g}_{\mathbf{1}}$ is a positive definite symmetric bilinear from on $\mathfrak{g}$ which is extended to $T \mathrm{G}$ by left translation:

$\mathbf{g}_{g}(g A, g B)=\mathbf{g}_{\mathbf{1}}(A, B) \quad$ for $\quad A, B \in \mathfrak{g}, g \in \mathrm{G}$.

Here, by the product $g A$ we mean $T_{1} L_{g} \cdot A$, where $L_{g}: h \mapsto g h$ is a left-translation. We recover a left-invariant Riemannian manifold if $\mathscr{D}=T \mathrm{G}$, i.e., $\mathscr{D}(\mathbf{1})=\mathfrak{g}$.

Remark. Right-invariant sub-Riemannian structures are defined similarly. Such structures are isometric to left-invariant ones (via Lie group anti-isomorphisms).

An absolutely continuous curve $g(\cdot):[0, T] \rightarrow \mathrm{G}$ is called a horizontal curve if $\dot{g}(t) \in \mathscr{D}(g(t))$ for almost all $t \in[0, T]$. We shall assume that $\mathscr{D}$ satisfies the bracket generating condition, i.e., $\mathscr{D}(\mathbf{1})$ generates $\mathfrak{g}$; this condition is necessary and sufficient for any two points in $\mathrm{G}$ to be connected by a horizontal curve. The length of a horizontal curve $g(\cdot)$ is given by

$\ell(g(\cdot))=\int_{0}^{T} \sqrt{\mathbf{g}(\dot{g}(t), \dot{g}(t))} d t$.

A sub-Riemannian manifold $(\mathrm{G}, \mathscr{D}, \mathbf{g})$ is endowed with a natural metric space structure, namely the Carnot-Carathéodory distance:

$d(g, h)=\inf \{\ell(g(\cdot)): g(\cdot)$ is a horizontal curve joining $g$ and $h\}$.

A horizontal curve $g(\cdot)$ that realizes the Carnot-Carathéodory distance between two points is called a minimising geodesic; these geodesics are fundamental objects of interest in the investigation of sub-Riemannian manifolds. Minimising geodesics exist between any two points if and only if the metric space $(\mathrm{G}, d)$ associated with Carnot-Carathéodory distance is complete ([16]).

By an isometry between two left-invariant sub-Riemannian (or Riemannian) manifolds $(\mathrm{G}, \mathscr{D}, \mathbf{g})$ and $\left(\mathrm{G}^{\prime}, \mathscr{D}^{\prime}, \mathbf{g}^{\prime}\right)$ we mean a diffeomorphism $\phi: \mathrm{G} \rightarrow \mathrm{G}^{\prime}$ such that

$\phi_{*} \mathscr{D}=\mathscr{D}^{\prime} \quad$ and $\quad \mathbf{g}=\phi^{*} \mathbf{g}^{\prime}$.

Any such isometry preserves the Carnot-Carathéodory distance in the sense that $d(g, h)=d^{\prime}(\phi(g), \phi(h))$. Isometries establish a one-to-one correspondence between the minimizing geodesics of $(\mathrm{G}, \mathscr{D}, \mathbf{g})$ and $\left(\mathrm{G}^{\prime}, \mathscr{D}^{\prime}, \mathbf{g}^{\prime}\right)$. 


\section{Automorphisms}

The automorphisms of $\mathfrak{h}_{n}$ are exactly those linear isomorphisms that preserve the center $\mathfrak{z}$ of $\mathfrak{h}_{n}$ and for which the induced map on $\mathfrak{h}_{n} / \mathfrak{z}$ preserves an appropriate symplectic structure (cf. [11]). More precisely, let $\Omega$ be the skew-symmetric bilinear form on $\mathfrak{h}_{n}$ specified by

$$
[A, B]=\Omega(A, B) Z, \quad A, B \in \mathfrak{h}_{n} .
$$

Note that $\Omega\left(X_{i}, Y_{j}\right)=\delta_{i j}$ and that $\Omega$ is zero on the remaining pairs of basis vectors. Accordingly, we get the following characterization of automorphisms.

Lemma 1. A linear isomorphism $\psi: \mathfrak{h}_{n} \rightarrow \mathfrak{h}_{n}$ is a Lie algebra automorphism if and only if

$\psi \cdot Z=c Z \quad$ and $\quad \Omega(\psi \cdot A, \psi \cdot B)=c \Omega(A, B)$

for some $c \neq 0$.

Proof. Suppose $\psi$ is an automorphism. It preserves the center of $\mathfrak{h}_{n}$ and therefore $\psi \cdot Z=c Z$ for some $c \neq 0$. For $A, B \in \mathfrak{h}_{n}$, we have

$\Omega(\psi \cdot A, \psi \cdot B) Z=\psi \cdot \Omega(A, B) Z$ and so $\Omega(\psi \cdot A, \psi \cdot B)=c \Omega(A, B)$.

Conversely, suppose $\psi$ is a linear isomorphism such that the given conditions hold. For $A, B \in \mathfrak{h}_{n}$, we have

$[\psi \cdot A, \psi \cdot B]=\Omega(\psi \cdot A, \psi \cdot B) Z=c \Omega(A, B) Z=\psi \cdot \Omega(A, B) Z=\psi \cdot[A, B]$.

Next, we give a matrix representation for the group of automorphisms. We shall make use of the ordered basis

$\left(Z, X_{1}, X_{2}, \ldots, X_{n}, Y_{1}, Y_{2}, \ldots, Y_{n}\right)$

The bilinear form $\Omega$ takes the form

$\Omega=\left[\begin{array}{ll}0 & 0 \\ 0 & J\end{array}\right], \quad$ where $\quad J=\left[\begin{array}{cc}0 & I_{n} \\ -I_{n} & 0\end{array}\right]$.

We denote by $\rho$ the involution

$\rho=\left[\begin{array}{ccc}-1 & 0 & 0 \\ 0 & 0 & I_{n} \\ 0 & I_{n} & 0\end{array}\right]$

which is clearly an automorphism.

Proposition 1 (cf. [17]). The group of automorphisms Aut $\left(\mathfrak{h}_{n}\right)$ is given by

$\left\{\left[\begin{array}{cc}r^{2} & \mathbf{v} \\ 0 & r g\end{array}\right], \rho\left[\begin{array}{cc}r^{2} & \mathbf{v} \\ 0 & r g\end{array}\right]: r>0, \mathbf{v} \in \mathbb{R}^{2 n}, g \in \operatorname{Sp}(n, \mathbb{R})\right\}$ 
where

$\operatorname{Sp}(n, \mathbb{R})=\left\{g \in \mathbb{R}^{2 n \times 2 n}: g^{\top} J g=J\right\}$

is the $n(2 n+1)$-dimensional symplectic group over $\mathbb{R}$.

Proof. It is easy to show (by use of the lemma) that the given maps are automorphisms. Suppose $\psi$ is an automorphism. Then $\psi \cdot Z=c Z$ for some $c \neq 0$. We assume $c>0$. (If $c<0$, then $\rho \psi$ is of the required form.) Thus

$\psi=\left[\begin{array}{cc}r^{2} & \mathbf{v} \\ 0 & M\end{array}\right]$

for some $r>0, \mathbf{v} \in \mathbb{R}^{2 n}$ and $M \in \mathrm{GL}(2 n, \mathbb{R})$. It then follows that $M^{\top} J M=r^{2} J$. For $g=\frac{1}{r} M$, we get $g^{\top} J g=J$. Thus

$\psi=\left[\begin{array}{cc}r^{2} & \mathbf{v} \\ 0 & r g\end{array}\right]$

for some $r>0, \mathbf{v} \in \mathbb{R}^{2 n}$ and $g \in \operatorname{Sp}(n, \mathbb{R})$.

Remark. Each automorphism decomposes as a (semidirect) product of

- a translation or inner automorphism $\left[\begin{array}{cc}1 & \mathbf{v} \\ 0 & I_{2 n}\end{array}\right], \mathbf{v} \in \mathbb{R}^{2 n}$

- a dilation $\left[\begin{array}{cc}r^{2} & 0 \\ 0 & r I_{2 n}\end{array}\right], r>0$

- a symplectic transformation $\left[\begin{array}{ll}1 & 0 \\ 0 & g\end{array}\right], g \in \operatorname{Sp}(n, \mathbb{R})$

- and possibly the involution $\rho$.

Indeed, we have the following decomposition as semidirect products:

$\operatorname{Aut}\left(\mathfrak{h}_{n}\right) \cong \mathbb{R}^{2 n} \rtimes \mathbb{R} \rtimes \mathrm{Sp}(n, \mathbb{R}) \rtimes\{\mathbf{1}, \rho\}$.

\section{Classification}

Diffeomorphisms that are compatible with the Lie group structure (in the sense that they preserve left-invariant vector fields) are automorphisms. For the purposes of this paper, we consider only those isometries that are also Lie group automorphisms. We shall refer to such isometries as $\mathfrak{L}$-isometries. For a given left-invariant subRiemannian manifold $(\mathrm{G}, \mathscr{D}, \mathbf{g})$ on a Carnot group $\mathrm{G}$, it turns out that the group of isometries $\phi:(\mathrm{G}, \mathscr{D}, \mathbf{g}) \rightarrow(\mathrm{G}, \mathscr{D}, \mathbf{g})$ decomposes as a semidirect product of the left translations (normal) and the $\mathfrak{L}$-isometries ([14]). We say that two left-invariant sub-Riemannian (resp. Riemannian) structures are $\mathfrak{L}$-isometric if there exists a $\mathfrak{L}$-isometry between them. We classify, under this equivalence relation, the leftinvariant sub-Riemannian and Riemannian manifolds on $\mathrm{H}_{n}$. By left invariance, we have the following simple characterization for $\mathfrak{L}$-isometries. 
Proposition 2. Suppose $\mathrm{G}$ and $\mathrm{G}^{\prime}$ are simply connected. $(\mathrm{G}, \mathscr{D}, \mathbf{g})$ and $\left(\mathrm{G}^{\prime}, \mathscr{D}^{\prime}, \mathbf{g}^{\prime}\right)$ are $\mathfrak{L}$-isometric if and only if there exists a Lie algebra isomorphism $\psi: \mathfrak{g} \rightarrow \mathfrak{g}^{\prime}$ such that $\psi \cdot \mathscr{D}(\mathbf{1})=\mathscr{D}^{\prime}(\mathbf{1})$ and $\mathbf{g}_{\mathbf{1}}(A, B)=\mathbf{g}_{\mathbf{1}}^{\prime}(\psi \cdot A, \psi \cdot B)$.

We consider the sub-Riemannian case first; we start by normalizing the distribution.

Lemma 2. For any (bracket-generating) left-invariant distribution $\mathscr{D}$ there exists an inner automorphism $\phi \in \operatorname{Aut}\left(\mathrm{H}_{n}\right)$ such that $\phi_{*} \mathscr{D}=\overline{\mathscr{D}}$, where $\overline{\mathscr{D}}$ is the leftinvariant distribution specified by $\overline{\mathscr{D}}(\mathbf{1})=\operatorname{span}\left(X_{1}, \ldots, X_{n}, Y_{1}, \ldots, Y_{n}\right)$.

Proof. It suffices to show that there exists a inner automorphism $\psi \in \operatorname{Aut}\left(\mathfrak{h}_{n}\right)$ such that $\psi \cdot \mathscr{D}(\mathbf{1})=\overline{\mathscr{D}}(\mathbf{1})$. For any subspace $\mathfrak{s} \subseteq \mathfrak{h}_{n}$, we have $\operatorname{Lie}(\mathfrak{s}) \leq \operatorname{span}(\mathfrak{s}, Z)$. Therefore, if $\operatorname{Lie}(\mathfrak{s})=\mathfrak{h}_{n}$ and $\mathfrak{s} \neq \mathfrak{h}_{n}$, then $\mathfrak{s}$ has codimension one and takes the form

$\mathfrak{s}=\operatorname{span}\left(X_{1}+v_{1} Z, \ldots, X_{n}+v_{n} Z, Y_{1}+v_{n+1} Z, \ldots, Y_{n}+v_{2 n} Z\right)$.

Accordingly,

$\psi=\left[\begin{array}{cc}1 & -\mathbf{v} \\ 0 & I_{2 n}\end{array}\right], \quad \mathbf{v}=\left[\begin{array}{llll}v_{1} & v_{2} & \cdots & v_{2 n}\end{array}\right]$

is an inner automorphism such that $\psi \cdot \mathfrak{s}=\operatorname{span}\left(X_{1}, \ldots, X_{n}, Y_{1}, \ldots, Y_{n}\right)$.

We now proceed to normalise the sub-Riemannian metric and so obtain a classification of the sub-Riemannian structures. We shall make use of Williamson's theorem, which states that positive definite matrices are diagonalizable by symplectic matrices (see [10], Chapter 8.3: "Symplectic Spectrum and Williamson's Theorem"). More precisely,

Lemma 3. If $M$ is a positive definite $2 n \times 2 n$ matrix, then there exists $S \in \operatorname{Sp}(n, \mathbb{R})$ such that

$S^{\top} M S=\left[\begin{array}{cc}\Lambda & 0 \\ 0 & \Lambda\end{array}\right], \quad \Lambda=\operatorname{diag}\left(\lambda_{1}, \lambda_{2}, \ldots, \lambda_{n}\right)$

where $\lambda_{1} \geq \lambda_{2} \geq \cdots \geq \lambda_{n}>0$.

The array $\operatorname{Spec}(M)=\left(\lambda_{1}, \ldots, \lambda_{n}\right)$ is called the symplectic spectrum of $M$. (The matrix $J M$ has eigenvalues values $\pm i \lambda_{j}$.) $\operatorname{Spec}(M)$ is a symplectic invariant, i.e., $\operatorname{Spec}\left(S^{\top} M S\right)=\operatorname{Spec}(M)$ for $S \in \operatorname{Sp}(n, \mathbb{R})$.

Theorem 1. Any left-invariant sub-Riemannian structure $(\mathscr{D}, \mathbf{g})$ on $\mathrm{H}_{n}$ is $\mathfrak{L}$-isometric to exactly one of the structures $\left(\overline{\mathscr{D}}, \overline{\mathbf{g}}^{\lambda}\right)$ specified by

$$
\left\{\begin{aligned}
\overline{\mathscr{D}}(\mathbf{1}) & =\operatorname{span}\left(X_{1}, \ldots, X_{n}, Y_{1}, \ldots, Y_{n}\right) \\
\overline{\mathbf{g}}_{\mathbf{1}}^{\lambda} & =\left[\begin{array}{lr}
\Lambda & 0 \\
0 & \Lambda
\end{array}\right], \quad \Lambda=\operatorname{diag}\left(\lambda_{1}, \lambda_{2}, \ldots, \lambda_{n}\right) .
\end{aligned}\right.
$$

Here $1=\lambda_{1} \geq \lambda_{2} \geq \cdots \geq \lambda_{n}>0$ parametrize a family of (non-equivalent) class representatives. 
Proof. By lemma 2, $(\mathscr{D}, \mathbf{g})$ is $\mathfrak{L}$-isometric to $\left(\overline{\mathscr{D}}, \mathbf{g}^{\prime}\right)$ for some left-invariant metric $\mathbf{g}^{\prime}$. The automorphisms

$$
\left[\begin{array}{cc}
r^{2} & 0 \\
0 & r I_{2 n}
\end{array}\right], r>0 \quad \text { and } \quad\left[\begin{array}{ll}
1 & 0 \\
0 & g
\end{array}\right], g \in \operatorname{Sp}(n, \mathbb{R})
$$

preserve the subspace $\overline{\mathscr{D}}(\mathbf{1})$, in the sense that $\psi \cdot \overline{\mathscr{D}}(\mathbf{1})=\overline{\mathscr{D}}(\mathbf{1})$. Let $Q$ be the matrix of the inner product $\mathbf{g}_{\mathbf{1}}^{\prime}$ on $\operatorname{span}\left(X_{1}, \ldots, X_{n}, Y_{1}, \ldots, Y_{n}\right)$. There exists $g \in \operatorname{Sp}(n, \mathbb{R})$ such that

$$
g^{\top} Q g=\left[\begin{array}{cc}
\Lambda & 0 \\
0 & \Lambda
\end{array}\right]
$$

where $\Lambda=\operatorname{diag}\left(\lambda_{1}, \ldots, \lambda_{n}\right)$ and $\left(\lambda_{1}, \ldots, \lambda_{n}\right)=\operatorname{Spec}(Q)$. Hence

$\left(\frac{1}{\sqrt{\lambda_{1}}} g\right)^{\top} Q\left(\frac{1}{\sqrt{\lambda_{1}}} g\right)=\left[\begin{array}{cc}\Lambda^{\prime} & 0 \\ 0 & \Lambda^{\prime}\end{array}\right]$

where $\Lambda^{\prime}=\operatorname{diag}\left(1, \frac{\lambda_{2}}{\lambda_{1}}, \ldots, \frac{\lambda_{n}}{\lambda_{1}}\right)$. Therefore

$\psi=\left[\begin{array}{ll}1 & 0 \\ 0 & g\end{array}\right]\left[\begin{array}{cc}\frac{1}{\lambda_{1}} & 0 \\ 0 & \frac{1}{\sqrt{\lambda_{1}}} I_{2 n}\end{array}\right]$

is an automorphism such that $\mathbf{g}_{\mathbf{1}}^{\prime \prime}(A, B)=\mathbf{g}_{\mathbf{1}}^{\prime}(\psi \cdot A, \psi \cdot B)$, where $\mathbf{g}_{\mathbf{1}}^{\prime \prime}$ has matrix $\left[\begin{array}{cc}\Lambda^{\prime} & 0 \\ 0 & \Lambda^{\prime}\end{array}\right]$. Consequently (relabelling $\frac{\lambda_{i}}{\lambda_{1}}$ as $\lambda_{i}$ ), the result follows by proposition 2 .

It remains to be shown that no two class representatives are equivalent. Suppose $\left(\overline{\mathscr{D}}, \overline{\mathbf{g}}^{\lambda}\right)$ and $\left(\overline{\mathscr{D}}, \overline{\mathbf{g}}^{\lambda^{\prime}}\right)$ are $\mathfrak{L}$-isometric, i.e., there exists an automorphism

$\psi=\left[\begin{array}{cc}r^{2} & \mathbf{v} \\ 0 & r g\end{array}\right] \quad$ or $\quad \psi=\rho\left[\begin{array}{cc}r^{2} & \mathbf{v} \\ 0 & r g\end{array}\right]$

such that $\psi \cdot \overline{\mathscr{D}}(\mathbf{1})=\overline{\mathscr{D}}(\mathbf{1})$ and $\overline{\mathbf{g}}_{\mathbf{1}}^{\lambda}(A, B)=\overline{\mathbf{g}}_{\mathbf{1}}^{\lambda^{\prime}}(\psi \cdot A, \psi \cdot B)$. The former condition implies $\mathbf{v}=0$ and so the latter implies $\Lambda=r^{2} g^{\top} \Lambda^{\prime} g$, where $\Lambda=\operatorname{diag}\left(\lambda_{1}, \ldots, \lambda_{n}\right)$ and $\Lambda^{\prime}=\operatorname{diag}\left(\lambda_{1}^{\prime}, \ldots, \lambda_{n}^{\prime}\right)$. Thus, by symplectic invariance, we have $\operatorname{Spec}(\Lambda)=$ $r^{2} \operatorname{Spec}\left(\Lambda^{\prime}\right)$. However for both $\operatorname{Spec}(\Lambda)$ and $\operatorname{Spec}\left(\Lambda^{\prime}\right)$, the dominant value is one; so $r=1$. Consequently $\Lambda=\Lambda^{\prime}$. That is to say, $\left(\overline{\mathscr{D}}, \overline{\mathbf{g}}^{\lambda}\right)$ and $\left(\overline{\mathscr{D}}, \overline{\mathbf{g}}^{\lambda^{\prime}}\right)$ are $\mathfrak{L}$ isometric only if $\lambda=\lambda^{\prime}$.

Corollary. Any left-invariant sub-Riemannian structure $(\mathscr{D}, \mathbf{g})$ on $\mathrm{H}_{n}$ is $\mathfrak{L}$-isometric to a structure with

$\left(v_{1} X_{1}, v_{2} X_{2}, \ldots, v_{n} X_{n}, v_{1} Y_{1}, v_{2} Y_{2}, \ldots, v_{n} Y_{n}\right)$

as orthonormal basis. Here $1=v_{1} \leq v_{2} \leq \ldots \leq v_{n}$ parametrize a family of (nonequivalent) class representatives.

We have the following coordinate-free version of Williamson's theorem. Let $\mu$ and $\mu^{\prime}$ be scalar products on a symplectic vector space $\left(\mathbb{R}^{2 n}, \omega\right)$. The symplectic spectrum of $\mu$ (resp. $\mu^{\prime}$ ) is the set of moduli of eigenvalues of the unique linear transformation $\kappa$ defined by $\omega(\mathbf{x}, \kappa \cdot \mathbf{y})=\mu(\mathbf{x}, \mathbf{y})$. A symplectic transformation is a linear isomorphism $\sigma$ such that $\omega(\sigma \cdot \mathbf{x}, \sigma \cdot \mathbf{y})=\omega(\mathbf{x}, \mathbf{y})$. 
Lemma 4. There exists a symplectic transformation $\sigma$ such that

$\mu(\mathbf{x}, \mathbf{y})=\mu^{\prime}(\sigma \cdot \mathbf{x}, \sigma \cdot \mathbf{y})$

if and only if the symplectic spectra of $\mu$ and $\mu^{\prime}$ are identical.

Proof. There exists a basis for $\mathbb{R}^{2 n}$ such that $\omega$ has matrix $J$. (A linear map $\sigma$ is then a symplectic transformation if and only if its matrix is a symplectic matrix.) Let $K$ and $M$ be the matrices of $\kappa$ and $\mu$, respectively. We have $K=-J M$. Hence the symplectic spectrum of $\mu$ is the same as the symplectic spectrum of $M$ (only, every value for $M$ is repeated twice for $\mu)$. If $\mu(\mathbf{x}, \mathbf{y})=\mu^{\prime}(\sigma \cdot \mathbf{x}, \sigma \cdot \mathbf{y})$, then $M=S^{\top} M^{\prime} S$ (here $S \in \mathrm{Sp}(n, \mathbb{R})$ is the matrix of $\sigma$ ) and so the symplectic spectra of $M$ and $M^{\prime}$ (resp. $\mu$ and $\mu^{\prime}$ ) match. Conversely, if $\mu$ and $\mu^{\prime}$ have identical symplectic spectra, then there exists symplectic matrices $S, S^{\prime} \in \mathrm{Sp}(n, \mathbb{R})$ such that $S^{\top} M S=S^{\prime^{\top}} M^{\prime} S^{\prime}$. Consequently, $M=\left(S^{\prime} S^{-1}\right)^{\top} M^{\prime}\left(S^{\prime} S^{-1}\right)$ and so $\mu(\mathbf{x}, \mathbf{y})=\mu^{\prime}(\sigma \cdot \mathbf{x}, \sigma \cdot \mathbf{y})$ where $\sigma$ is the unique symplectic transformation with matrix $S^{\prime} S^{-1}$.

The Lie algebra $\mathfrak{h}_{n}$ (as a vector space) can be decomposed as the direct sum of a symplectic vector space $\left(\mathbb{R}^{2 n}, \omega\right)$ and $\mathbb{R}$; the Lie bracket of two elements is given by

$[(\mathbf{v}, z),(\mathbf{v}, z)]=\left(0, \omega\left(\mathbf{v}, \mathbf{v}^{\prime}\right)\right) \quad$ for $\quad(\mathbf{v}, z),\left(\mathbf{v}^{\prime}, z\right) \in \mathbb{R}^{2 n} \oplus \mathbb{R}$.

By lemma 2, any sub-Riemannian structure $(\mathscr{D}, \mathbf{g})$ is $\mathfrak{L}$-isometric to one for which $\mathscr{D}(\mathbf{1})=\mathbb{R}^{2 n}$. Hence the metric $\mathbf{g}_{\mathbf{1}}$ is a scalar product on $\mathbb{R}^{2 n}$. The normalized symplectic spectrum of a scalar product is the symplectic spectrum normalized by the dominant value: $\left\{1, \frac{\lambda_{2}}{\lambda_{1}}, \frac{\lambda_{3}}{\lambda_{1}}, \ldots, \frac{\lambda_{n}}{\lambda_{1}}\right\}$. Accordingly, by the foregoing considerations, we get the following coordinate-free characterization of the sub-Riemannian structures.

Theorem 2. Suppose $(\mathscr{D}, \mathbf{g})$ and $\left(\mathscr{D}^{\prime}, \mathbf{g}^{\prime}\right)$ are two left-invariant sub-Riemannian structures on $\mathrm{H}_{n}$ such that $\mathscr{D}(\mathbf{1})=\mathscr{D}^{\prime}(\mathbf{1})=\mathbb{R}^{2 n}$. Then $(\mathscr{D}, \mathbf{g})$ and $\left(\mathscr{D}^{\prime}, \mathbf{g}^{\prime}\right)$ are $\mathfrak{L}$ isometric if and only if the normalized symplectic spectra of $\mathbf{g}_{\mathbf{1}}$ and $\mathbf{g}_{\mathbf{1}}^{\prime}$ are identical.

Next, we consider the Riemannian case; the classification result is similar to the sub-Riemannian case.

Theorem 3. Any left-invariant Riemannian structure $\mathbf{g}$ on $\mathrm{H}_{n}$ is $\mathfrak{L}$-isometric to exactly one of the structures

$\overline{\mathbf{g}}_{1}^{\lambda}=\left[\begin{array}{ccc}1 & 0 & 0 \\ 0 & \Lambda & 0 \\ 0 & 0 & \Lambda\end{array}\right], \quad \Lambda=\operatorname{diag}\left(\lambda_{1}, \lambda_{2}, \ldots, \lambda_{n}\right)$.

Here $\lambda_{1} \geq \lambda_{2} \geq \cdots \geq \lambda_{n}>0$ parametrize a family of (non-equivalent) class representatives.

Proof. Let $R$ be the matrix of the inner product $\mathbf{g}_{1}$ on $\mathfrak{h}_{n}$. We have

$R=\left[\begin{array}{ll}\frac{1}{r^{4}} & \mathbf{v} \\ \mathbf{v}^{\top} & Q\end{array}\right]$ 
for some $r>0, \mathbf{v} \in \mathbb{R}^{2 n}$ and $Q \in \mathbb{R}^{2 n \times 2 n}$. Hence we get

$\psi=\left[\begin{array}{cc}r^{2} & -r^{5} \mathbf{v} \\ 0 & r I_{2 n}\end{array}\right] \in \operatorname{Aut}\left(\mathfrak{h}_{n}\right) \quad$ and $\quad \psi^{\top} R \psi=\left[\begin{array}{cc}1 & 0 \\ 0 & Q^{\prime}\end{array}\right]$

for some positive definite matrix $Q^{\prime}$. Accordingly, there exists an automorphism $\psi^{\prime}=\left[\begin{array}{ll}1 & 0 \\ 0 & g\end{array}\right], g \in \mathrm{Sp}(n, \mathbb{R})$ such that

$\left(\psi \circ \psi^{\prime}\right)^{\top} R\left(\psi \circ \psi^{\prime}\right)=\left[\begin{array}{ccc}1 & 0 & 0 \\ 0 & \Lambda & 0 \\ 0 & 0 & \Lambda\end{array}\right]$

where $\Lambda=\operatorname{diag}\left(\lambda_{1}, \ldots, \lambda_{n}\right)$ and $\left(\lambda_{1}, \ldots, \lambda_{n}\right)=\operatorname{Spec}\left(Q^{\prime}\right)$. Consequently, the result follows by proposition 2 . As in the sub-Riemannian case, it is a simple matter to show that none of these structures are $\mathfrak{L}$-isometric.

Corollary. Any left-invariant Riemannian structure $\mathbf{g}$ on $\mathrm{H}_{n}$ is $\mathfrak{L}$-isometric to a structure with

$\left(Z, v_{1} X_{1}, v_{2} X_{2}, \ldots, v_{n} X_{n}, v_{1} Y_{1}, v_{2} Y_{2}, \ldots, v_{n} Y_{n}\right)$

as orthonormal basis. Here $0<v_{1} \leq v_{2} \leq \cdots \leq v_{n}$ parametrize a family of (nonequivalent) class representatives.

Any Riemannian structure on $\mathrm{H}_{n}$ is $\mathfrak{L}$-isometric to one for which the scalar product $\mathbf{g}_{1}$ on $\left(\mathbb{R}^{2 n} \oplus \mathbb{R}\right)$ decomposes as

$\mathbf{g}_{\mathbf{1}}\left((\mathbf{v}, z),\left(\mathbf{v}^{\prime}, z^{\prime}\right)\right)=\mu_{\mathbf{g}}\left(\mathbf{v}, \mathbf{v}^{\prime}\right)+z z^{\prime}$

where $\mu_{\mathrm{g}}$ is a scalar product on $\mathbb{R}^{2 n}$. Accordingly, we have the following coordinatefree characterization the Riemannian structures.

Theorem 4. Suppose $\mathbf{g}$ and $\mathbf{g}^{\prime}$ define two left-invariant Riemannian structures on $\mathrm{H}_{n}$ such that

$\mathbf{g}_{\mathbf{1}}\left((\mathbf{v}, z),\left(\mathbf{v}^{\prime}, z^{\prime}\right)\right)=\mu_{\mathbf{g}}\left(\mathbf{v}, \mathbf{v}^{\prime}\right)+z z^{\prime} \quad$ and $\quad \mathbf{g}_{\mathbf{1}}\left((\mathbf{v}, z),\left(\mathbf{v}^{\prime}, z^{\prime}\right)\right)=\mu_{\mathbf{g}^{\prime}}\left(\mathbf{v}, \mathbf{v}^{\prime}\right)+z z^{\prime}$.

Then $\mathbf{g}$ and $\mathbf{g}^{\prime}$ are $\mathfrak{L}$-isometric if and only if the symplectic spectra of $\mu_{\mathbf{g}}$ and $\mu_{\mathbf{g}^{\prime}}$ are identical.

\section{Invariant Optimal Control}

Invariant control systems on Lie groups were first considered in 1972 by Brockett [6] and by Jurdjevic and Sussmann [13]. A left-invariant control affine system on a (real, finite-dimensional) Lie group $G$ is a collection of left-invariant vector fields $\Xi(\cdot, u)$ on $G$, affinely parametrized by controls. In classical notation, a drift-free system $\Sigma=(\mathrm{G}, \Xi)$ is written as

$\dot{g}=g \Xi(\mathbf{1}, u)=g\left(u_{1} B_{1}+\cdots+u_{\ell} B_{\ell}\right), \quad g \in \mathrm{G}, u \in \mathbb{R}^{\ell}$. 
Here the parametrization map $\Xi(\mathbf{1}, \cdot): \mathbb{R}^{\ell} \rightarrow \mathfrak{g}$ is an injective affine map (i.e., $B_{1}, \ldots, B_{\ell}$ are linearly independent). The "product" $g \Xi(\mathbf{1}, u)$ is given by $g \Xi(\mathbf{1}, u)=$ $T_{1} L_{g} \cdot \Xi(\mathbf{1}, u)$, where $L_{g}: \mathrm{G} \rightarrow \mathrm{G}, h \mapsto g h$ is the left translation by $g$. The dynamics $\Xi: G \times \mathbb{R}^{\ell} \rightarrow T G$ are invariant under left translations, i.e., $\Xi(g, u)=g \Xi(\mathbf{1}, u)$. An admissible control is a piecewise continuous map $u(\cdot):[0, T] \rightarrow \mathbb{R}^{\ell}$. A trajectory corresponding to an admissible control $u(\cdot)$ is a absolutely continuous curve $g(\cdot):[0, T] \rightarrow \mathrm{G}$ such that $\dot{g}(t)=\Xi(g(t), u(t))$ almost everywhere. A system is said to be controllable if any two states can be joined by a trajectory. For more details about invariant control systems see, e.g., $[13,18,2,12]$.

An invariant optimal control problem is defined by the specification of $(i)$ a leftinvariant control system, (ii) a positive definite quadratic cost function $L: \mathbb{R}^{\ell} \rightarrow$ $\mathbb{R}$ and (iii) boundary data, consisting of an initial state $g_{0} \in \mathrm{G}$, a terminal state $g_{1} \in \mathrm{G}$ and a (fixed) terminal time $T>0$. Explicitly, we wish to minimize the functional $\mathscr{J}=\int_{0}^{T} L(u(t)) d t$ over trajectory-control pairs, subject to the boundary data $g(0)=g_{0}, \quad g(T)=g_{1}$. We associate to such a problem, the cost-extended system $(\Sigma, L)$ consisting of a controllable system $\Sigma$ and a cost function $L$. Two cost-extended systems $(\Sigma=(\mathrm{G}, \Xi), L)$ and $\left(\Sigma^{\prime}=\left(\mathrm{G}^{\prime}, \Xi^{\prime}\right), L^{\prime}\right)$ are cost-equivalent ([3]) if there exist a Lie group isomorphism $\phi: G \rightarrow G^{\prime}$ and a linear isomorphism $\varphi: \mathbb{R}^{\ell} \rightarrow \mathbb{R}^{\ell}$ such that

$T_{g} \phi \cdot \Xi(g, u)=\Xi^{\prime}(\phi(g), \varphi(u)) \quad$ and $\quad r L=L^{\prime} \circ \varphi$

for some $r>0$. The automorphism $\phi$ establishes a one-to-one correspondence between the optimal trajectories (or corresponding minimising geodesics) of $(\Sigma, L)$ and $\left(\Sigma^{\prime}, L^{\prime}\right)$. By left invariance, we have that $(\Sigma, L)$ and $\left(\Sigma^{\prime}, L^{\prime}\right)$ are cost-equivalent if and only if there exist a Lie group isomorphism $\phi: G \rightarrow G^{\prime}$ and an affine isomorphism $\varphi: \mathbb{R}^{\ell} \rightarrow \mathbb{R}^{\ell^{\prime}}$ such that $T_{\mathbf{1}} \phi \cdot \Xi(\mathbf{1}, u)=\Xi^{\prime}\left(\mathbf{1}^{\prime}, \varphi(u)\right)$ and $L^{\prime} \circ \varphi=r L$ for some $r>0$.

Analogous to theorems 1 and 3, we get the following classification of cost-extended systems on $\mathrm{H}_{n}$.

Theorem 5. Any cost-extended system on $\mathrm{H}_{n}$ is cost-equivalent to exactly one of the following cost-extended systems:

$$
\begin{array}{ll}
\left(\Sigma^{2 n}, L_{\lambda}^{2 n}\right): & \left\{\begin{array}{l}
\Xi^{2 n}(\mathbf{1}, u)=\sum_{i=1}^{n}\left(u_{X_{i}} X_{i}+u_{Y_{i}} Y_{i}\right) \\
L_{\lambda}^{2 n}(u)=\sum_{i=1}^{n} \lambda_{i}\left(u_{X_{i}}^{2}+u_{Y_{i}}^{2}\right)
\end{array}\right. \\
\left(\Sigma^{2 n+1}, L_{\lambda}^{2 n+1}\right): & \left\{\begin{array}{l}
\Xi^{2 n+1}(\mathbf{1}, u)=u_{Z} Z+\sum_{i=1}^{n}\left(u_{X_{i}} X_{i}+u_{Y_{i}} Y_{i}\right) \\
L_{\lambda}^{2 n+1}(u)=u_{Z}^{2}+\sum_{i=1}^{n} \lambda_{i}\left(u_{X_{i}}^{2}+u_{Y_{i}}^{2}\right) .
\end{array}\right.
\end{array}
$$

Here $1=\lambda_{1} \geq \lambda_{2} \geq \cdots \geq \lambda_{n}>0$ parametrize families of (non-equivalent) class representatives. 
Remark. The associated optimal control problems are:

$$
\begin{aligned}
& \left\{\begin{array}{l}
\dot{g}=g \sum_{i=1}^{n}\left(u_{X_{i}} X_{i}+u_{Y_{i}} Y_{i}\right), \quad g \in \mathrm{H}_{n},\left(u_{X_{1}}, \ldots, u_{Y_{n}}\right) \in \mathbb{R}^{2 n} \\
g(0)=g_{0} \quad g(T)=g_{1} \\
\int_{0}^{T} \sum_{i=1}^{n} \lambda_{i}\left(u_{X_{i}}(t)^{2}+u_{Y_{i}}(t)^{2}\right) d t \longrightarrow \min
\end{array}\right. \\
& \left\{\begin{array}{l}
\dot{g}=g\left(u_{Z} Z+\sum_{i=1}^{n}\left(u_{X_{i}} X_{i}+u_{Y_{i}} Y_{i}\right)\right), \quad g \in \mathrm{H}_{n},\left(u_{Z}, u_{X_{1}}, \ldots, u_{Y_{n}}\right) \in \mathbb{R}^{2 n+1} \\
g(0)=g_{0} \quad g(T)=g_{1} \\
\int_{0}^{T}\left(u_{Z}(t)^{2}+\sum_{i=1}^{n} \lambda_{i}\left(u_{X_{i}}(t)^{2}+u_{Y_{i}}(t)^{2}\right)\right) d t \longrightarrow \text { min. }
\end{array}\right.
\end{aligned}
$$

Solutions of these optimal control problems are minimising geodesics for the corresponding sub-Riemannian (resp. Riemannian) structures.

\section{Conclusions}

We have obtained an explicit classification of the sub-Riemannian (and Riemannian) structures on $\mathrm{H}_{n}$; an analogous classification of cost-extended control systems was also exhibited. In particular, we found that the Riemannian structures on $\mathrm{H}_{n}$ can be parametrized (up to an $\mathfrak{L}$-isometry) by $n$ parameters, whereas the sub-Riemannian structures can be parametrized by $n-1$ parameters. Agrachev and Barilari [1] classified the invariant sub-Riemannian structures on three-dimensional Lie groups; in particular, they show that all left-invariant sub-Riemannian structures on $\mathrm{H}_{1}$ are locally isometric. We have shown that all left-invariant sub-Riemannian structures on $\mathrm{H}_{1}$ are in fact (globally) isometric. Topics for future research include the calculation of the isometry groups and geodesics as well as extensions to Finsler structures.

\section{Acknowledgement}

The research leading to these results has received funding from the European Union's Seventh Framework Programme (FP7/2007-2013) under grant agreement no. 317721. Also, the first author would like to acknowledge the financial assistance of the National Research Foundation (DAAD-NRF) and Rhodes University towards this research.

\section{References}

[1] A. Agrachev and D. Barilari, Sub-Riemannian structures on 3D Lie groups, J. Dynam. Control Syst. 18 (2012), 21-44

[2] A.A. Agrachev and Y.L. Sachkov, Control Theory from the Geometric Viewpoint, Springer-Verlag, 2004

[3] R. Biggs and C.C. Remsing, On the equivalence of cost-extended control systems on Lie groups, Proc. 8th WSEAS Int. Conf. Dyn. Syst. \& Control, Porto, Portugal (2012), 60-65 
[4] J. Berndt, F. Tricceri and L. Vanhecke, Generalized Heisenberg Groups and Damek-Ricci Harmonic Spaces, Lecture Notes in Math. Vol. 1598, SpringerVerlag, 1995

[5] A.M. Bloch, Nonholonomic Mechanics and Control, Springer-Verlag, 2003

[6] R.W. Brockett, System theory on group manifolds and coset spaces, SIAM J. Control 10 (1972), 265-284

[7] O. Calin and D.-C. Chang, Sub-Riemannian Geometry: General Theory and Examples, Cambridge University Press, 2009

[8] O. Calin, D.-C. Chang and S.S.T. Yau, Nonholonomic systems and subRiemannian geometry, Commun. Inf. Syst. 10 (2010), 293-316

[9] P. Eberlein, Geometry of 2-step nilpotent Lie groups, in Modern Dynamical Systems and Applications, ed. by M. Brin, B. Hasselblatt and Y. Pesin, Cambridge Universtiy Press, 2004, pp. 67-101

[10] M. de Gosson, Symplectic Geometry and Quantum Mechanics, volume 166 of Operator Theory: Advances and Applications, Birkhäuser, 2006

[11] V. V. Gorbatsevich, A. L. Onishchik, and E. B. Vinberg. Foundations of Lie theory and Lie transformation groups, Springer-Verlag, 1997

[12] V. Jurdjevic, Geometric Control Theory, Cambridge University Press, 1997

[13] V. Jurdjevic and H.J. Sussmann, Control systems on Lie groups, J. Diff. Equations 12 (1972), 313-329

[14] I. Kishimoto, Geodesics and isometries of Carnot groups, J. Math. Kyoto Univ. 43 (2003), 509-522

[15] J. Lauret, Homogeneous nilmanifolds attached to representations of compact Lie groups, Manuscripta Math. 99 (1999), 287-309

[16] R. Montgomery, A Tour of Subriemannian Geometries, Their Geodesics and Applications, American Mathematical Society, 2002

[17] L. Saal, The automorphism group of a Lie algebra of Heisenberg type, Rend. Sem. Mat. Univ. Politec. Torino 54 (1996), 101-113

[18] Y.L. Sachkov, Control theory on Lie groups, J. Math. Sci. 156 (2009), 381439

[19] K.-H. Tan and X.-P. Yang, Characterisation of the sub-Riemannian isometry groups of $H$-type groups, Bull. Austral. Math. Soc. 70 (2004), 87-100 